\title{
Stability estimates for the fault inverse problem
}

\author{
Faouzi Triki, ${ }^{*} \quad$ Darko Volkov ${ }^{\dagger}$
}

July 3, 2021

\section{Contents}

1 Introduction 2

2 Mathematical model and uniqueness result 3

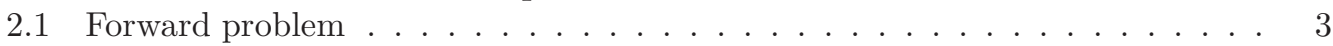

2.2 Fault inverse problem $\ldots \ldots \ldots \ldots \ldots \ldots$

3 Lipschitz stability of the fault geometry for a fixed slip 5

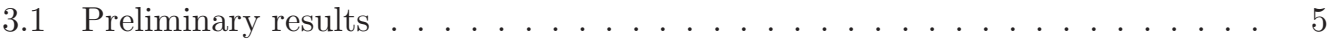

3.2 Lipschitz stability theorem for a fixed slip . . . . . . . . . . . 7

4 Second stability theorem: the case of unknown slips 12

5 Appendix: proof of Lemma 3.1

6 Conclusion 18

\begin{abstract}
We study in this paper stability estimates for the fault inverse problem. In this problem, faults are assumed to be planar open surfaces in a half space elastic medium with known Lamé coefficients. A traction free condition is imposed on the boundary of the half space. Displacement fields present jumps across faults, called slips, while traction derivatives are continuous. It was proved in 22 that if the displacement field is known on an open set on the boundary of the half space, then the fault and the slip are uniquely determined. In this present paper, we study the stability of this uniqueness result with regard to the coefficients of the equation of the plane containing the fault. If the slip field is known we state and prove a Lipschitz stability result. In the more interesting case where the slip field is unknown, we state and prove another Lipschitz stability result under the additional assumption, which is still physically relevant, that the slip field is one directional.
\end{abstract}

*Laboratoire Jean Kuntzmann, UMR CNRS 5224, Université Grenoble-Alpes, 700 Avenue Centrale, 38401 Saint-Martin-d'Hères, France. Email: faouzi.triki@univ-grenoble-alpes.fr.

${ }^{\dagger}$ Department of Mathematical Sciences, Worcester Polytechnic Institute, Worcester, MA 01609. Email: darko@wpi.edu. 


\section{Introduction}

Understanding and mapping the structure of Earth's crust in ever finer details has always captured the interest of geophysicists. Seismic and displacements data are collected by sensors and then processed using Partial Differential Equations (PDE) models and inverse problem formulations. Typical models for the Earth's crust involve linear elasticity equations: this is because displacements and deformations are very small compared to the thickness of the crust. Moreover, if local phenomena such as earthquakes or active subduction zones are studied, a half space formulation is adequate [3, 9, 11. With the advent of ultra accurate satellite based measurements of surface displacements (2 to 5 millimeter resolution) the study of so called "slow earthquakes" [5, 6, 10, 8, 15, 16] has recently attracted a lot of attention. Most authors first set a profile for the interface between tectonic plates (also called faults) derived from seismicity or gravimetry as in [13] and then use a linear inverse algorithm for determining slip fields on faults. A popular algorithm is the one explained in Tarantola's textbook [17. In addition to recovering these slip fields from surface displacement measurements, some authors have sought to simultaneously recover some geometric features of the fault, such as the dip angle [3, 9. However, until recently, there was no formal mathematical proof that the simultaneous recovery of the (piecewise linear) geometry of the fault and the slip was at all possible. This was achieved in [22. From there the second author and Sandmunienge have derived a deterministic and a stochastic fault reconstruction algorithm [21] and estimated convergence rates to the solution of the inverse problem. In 21, these convergence rates still depend on the intrinsic stability of the underlying inverse problem. Although numerical investigations hinted at a possible Lipschitz type stability, these stability estimates were still unknown at the time of writing of [21, and they are the subject of this present study. In general, a uniqueness statement for solving an inverse problem is not of great practical use without a stability result. From a pragmatic and computational point of view, mathematical objects can only be computed approximately and real life field data is always tinted by measurement errors, so one would not want these errors to grow exponentially in inversion algorithms.

A literature review of the field of inverse problems will show that stability results are notoriously difficult to derive and prove. The major difficulty in proving such results is that solutions to inverse problems are not explicitly formulated. There are a few papers on stability estimates for the recovery of cracks in materials, which is the analog of faults in Earth's crust. In an earlier paper, Friedman and Vogelius [7 showed a stability result for the recovery of liner cracks. In that paper the governing equation was the two dimensional conductivity and outer boundary conditions were prescribed to adequate values. In [1, Alessandrini et al. proved a general $\log \log$ stability estimate for the Hausdorff distance between two $C^{1,1}$ domains where in each domain there is a solution to the same conductivity equation with same Neumann condition and the stability estimate is in the $L^{2}$ distance between the corresponding Dirichlet outputs on one part of the boundary. In [2, Ammari and the first author were able to improve this $\log \log$ estimate based on the assumptions that Dirichet data is available for a whole range of frequencies and boundaries are a priori known to be open real analytic curves. In 4, Beretta et al. were also able to derive and prove an interesting Lipschitz stability result. Their result pertains to two dimensional linear elasticity in bounded domains with cracks. In the case of linear cracks they were able to derive Lipschitz continuity of the Hausdorff distance between cracks in terms of overdetermined boundary data. Here we need to explain that the case of faults which pertains to 
our research project is drastically different since we can not impose boundary conditions, we are simply passively measuring displacements on one part of the boundary of an infinite domain while an unknown slip field on an unknown fault is the forcing term of our governing equations.

This paper is organized as follows. In section 2 we introduce the PDE for the forward fault problem and we recall the uniqueness statement for the inverse fault problem proved in 22. Section 3 contains our first stability result and its proof. It relies on the implicit function theorem. Indeed, using the Green's function for the forward PDE, solutions can represented by convolution with the slip on the fault. We thus define a fault to surface operator. At fixed slip, in effect, this introduces a $C^{1}$ function $\phi$ from the set of geometry parameters $m$ in $\mathbb{R}^{3}$ defining the plane containing the fault to the space of surface measurements. We know from 22 that $\phi$ is injective. Thus, by the inverse function Theorem, if $\nabla \phi(m)$ has full rank, $\phi^{-1}$ is $C^{1}$ in a neighborhood of $\phi(m)$ and is therefore Lipschitz continuous. The crux of the proof is in proving that $\nabla \phi(m)$ has full rank. This is established by an argument by contradiction. If $\nabla \phi(m)$ does not have full rank, then using relations on jumps for the Green's function of the forward problem (and of its derivatives), we can derive a PDE for the slip field $\boldsymbol{h}$ on the fault. Finally, we prove that this PDE can only have the trivial solution, completing the proof. It turns out that although the PDE on $\boldsymbol{h}$ is a relatively simple transport equation in most cases, in the particular case of horizontal faults a much more complicated system of PDE for $\boldsymbol{h}$ must be solved. In section 4, we assume that a fixed but unknown slip $\boldsymbol{h}_{0}$ is occurring on a plane with geometry parameter $m_{0}$. Thus in this case it is not possible to evaluate the difference $\left\|\phi(m)-\phi\left(m_{0}\right)\right\|$. Instead, we use a linear operator $A_{m}$ mapping any slip $\phi$ to surface measurements $A_{m} \phi$ and we may minimize inf $\left\|A_{m} \phi-A_{m_{0}} \boldsymbol{h}_{0}\right\|$ where the inf is taken over all possible slips. This quantity is proven to be bounded below by a constant times $\left|m-m_{0}\right|$ under the additional assumption that $\boldsymbol{h}_{0}$ is one directional (that direction is not known for the inverse problem), or that $\boldsymbol{h}_{0}$ is a gradient. Finally, the rather technical formulas for the jumps of integrals containing a convolution of the elasticity Green's tensor with a vector field density are shown in Appendix. Although a related formula is standard in solid mechanics, we have not found formulas for the jumps of the first and second derivatives in the literature. This is probably due to the fact that they are not directly related to a physical problem and that they may be too intricate to prove without the use of symbolic computation software.

\section{Mathematical model and uniqueness result}

\subsection{Forward problem}

Using the standard rectangular coordinates $\boldsymbol{x}=\left(x_{1}, x_{2}, x_{3}\right)$ of $\mathbb{R}^{3}$, we define $\mathbb{R}^{3-}$ to be the open half space $x_{3}<0$. Let $\partial_{i}$ denote the derivative in the $i$-th coordinate. In this paper we consider the case of linear, homogeneous, isotropic elasticity; the two Lamé constants $\lambda$ and $\mu$ will be two positive constants. For a vector field $\boldsymbol{u}=\left(u_{1}, u_{2}, u_{3}\right)$ the stress and strain tensors will be denoted as follows,

$$
\begin{array}{r}
\sigma_{i j}(\boldsymbol{u})=\lambda \operatorname{div} \boldsymbol{u} \delta_{i j}+\mu\left(\partial_{i} u_{j}+\partial_{j} u_{i}\right), \\
\epsilon_{i j}(\boldsymbol{u})=\frac{1}{2}\left(\partial_{i} u_{j}+\partial_{j} u_{i}\right),
\end{array}
$$


and the stress vector in the direction $\boldsymbol{e} \in \mathbb{R}^{3}$ will be denoted by

$$
T_{e} \boldsymbol{u}=\sigma(\boldsymbol{u}) \boldsymbol{e} .
$$

Let $\Gamma$ be a Lipschitz open surface which is strictly included in $\mathbb{R}^{3-}$, with a normal vector $\boldsymbol{n}$. We define the jump $[\boldsymbol{v}]$ of a vector field $\boldsymbol{v}$ across $\Gamma$ to be

$$
[\boldsymbol{v}](\boldsymbol{x})=\lim _{h \rightarrow 0^{+}} \boldsymbol{v}(\boldsymbol{x}+h \boldsymbol{n})-\boldsymbol{v}(\boldsymbol{x}-h \boldsymbol{n}),
$$

for $\boldsymbol{x}$ in $\Gamma$, if this limit exists. Let $\boldsymbol{u}$ be the displacement field solving

$$
\begin{array}{r}
\mu \Delta \boldsymbol{u}+(\lambda+\mu) \nabla \operatorname{div} \boldsymbol{u}=0 \text { in } \mathbb{R}^{3-} \backslash \Gamma, \\
T_{\boldsymbol{e}_{3}} \boldsymbol{u}=0 \text { on the surface } x_{3}=0, \\
T_{\boldsymbol{n}} \boldsymbol{u} \text { is continuous across } \Gamma, \\
{[\boldsymbol{u}]=\boldsymbol{g} \text { is a given jump across } \Gamma,} \\
\boldsymbol{u}(\boldsymbol{x})=O\left(\frac{1}{|\boldsymbol{x}|^{2}}\right), \nabla \boldsymbol{u}(\boldsymbol{x})=O\left(\frac{1}{|\boldsymbol{x}|^{3}}\right), \text { uniformly as }|\boldsymbol{x}| \rightarrow \infty,
\end{array}
$$

where $\boldsymbol{e}_{3}$ is the vector $(0,0,1)$. For vector fields $\boldsymbol{v}, \boldsymbol{w}$ in $\mathbb{R}^{3-} \backslash \bar{\Gamma}$ whose gradient is square integrable we introduce the bilinear product

$$
B(\boldsymbol{v}, \boldsymbol{w})=\int_{\mathbb{R}^{3-} \backslash \bar{\Gamma}} \lambda \operatorname{tr}(\nabla \boldsymbol{v}) \operatorname{tr}(\nabla \boldsymbol{w})+2 \mu \operatorname{tr}(\epsilon(\boldsymbol{v}) \epsilon(\boldsymbol{w})),
$$

where tr is the trace. In [22], we defined the functional space $\mathcal{V}$ of vector fields $\boldsymbol{v}$ defined in $\mathbb{R}^{3-} \backslash \bar{\Gamma}$ such that $\nabla \boldsymbol{v}$ and $\frac{\boldsymbol{v}}{\left(1+r^{2}\right)^{\frac{1}{2}}}$ are in $L^{2}\left(\mathbb{R}^{3-} \backslash \bar{\Gamma}\right)$, and we proved that the following four norms are equivalent on $\mathcal{V}$ :

$$
\begin{array}{r}
\|\boldsymbol{v}\|_{1}=\left(\int_{\mathbb{R}^{3-} \backslash \bar{\Gamma}}|\nabla \boldsymbol{v}|^{2}\right)^{\frac{1}{2}}+\left(\int_{\mathbb{R}^{3-} \backslash \bar{\Gamma}} \frac{|\boldsymbol{v}|^{2}}{1+r^{2}}\right)^{\frac{1}{2}}, \\
\|\boldsymbol{v}\|_{2}=\left(\int_{\mathbb{R}^{3-} \backslash \bar{\Gamma}}|\nabla \boldsymbol{v}|^{2}\right)^{\frac{1}{2}}, \\
\|\boldsymbol{v}\|_{3}=\left(\int_{\mathbb{R}^{3-} \backslash \bar{\Gamma}}|\epsilon(\boldsymbol{v})|^{2}\right)^{\frac{1}{2}}, \\
\|\boldsymbol{v}\|_{4}=B(\boldsymbol{v}, \boldsymbol{v})^{1 / 2} .
\end{array}
$$

Let $D$ be a bounded domain with a Lipschitz boundary $\partial D$ containing $\Gamma$. We define the Sobolev space $\widetilde{H}^{\frac{1}{2}}(\Gamma)^{2}$ to be the set of restrictions to $\Gamma$ of tangential fields in $H^{\frac{1}{2}}(\partial D)^{2}$ supported in $\Gamma$. We proved in [22] the following theorem.

Theorem 2.1 Let $\boldsymbol{g}$ be in $\widetilde{H}^{\frac{1}{2}}(\Gamma)^{2}$. The problem 2.1 2.4 has a unique solution in $\mathcal{V}$. In addition the solution $\boldsymbol{u}$ satisfies the decay conditions 2.5.).

In this paper we will only consider forcing terms $\boldsymbol{g}$ which are tangential to $\Gamma$. Physically, this suggests that the fault $\Gamma$ is not opening or starting to self intersect: only slip is allowed. We recall that if $\boldsymbol{g}$ is continuous, the support of $\boldsymbol{g}, \operatorname{supp} \boldsymbol{g}$, is equal to the closure of the set of points in $\Gamma$ where $\boldsymbol{g}$ is non zero; in general supp $\boldsymbol{g}$ is defined in the sense of distributions. 


\subsection{Fault inverse problem}

Can we determine both $\boldsymbol{g}$ and $\Gamma$ from the data $\boldsymbol{u}$ given only on the plane $x_{3}=0$ ? The following Theorem in 22 asserts that this is possible if the data is known on a relatively open set of the plane $x_{3}=0$.

Theorem 2.2 Let $\Gamma_{1}$ and $\Gamma_{2}$ be two bounded open surfaces, with smooth boundary, such that each of them is included in a rectangle strictly contained in $\mathbb{R}^{3-}$. For $i$ in $\{1,2\}$, assume that $\boldsymbol{u}^{i}$ solves 2.1 2.5) for $\Gamma_{i}$ in place of $\Gamma$ and $\boldsymbol{g}^{i}$, a tangential field in $H_{0}^{1}\left(\Gamma_{i}\right)^{2}$, in place of $\boldsymbol{g}$. Assume that $\boldsymbol{g}^{i}$ has full support in $\Gamma_{i}$, that is, supp $\boldsymbol{g}_{i}=\overline{\Gamma_{i}}$. Let $V$ be a non empty open subset in $\left\{x_{3}=0\right\}$. If $\boldsymbol{u}^{1}$ and $\boldsymbol{u}^{2}$ are equal in $V$, then $\Gamma_{1}=\Gamma_{2}$ and $\boldsymbol{g}^{1}=\boldsymbol{g}^{2}$.

The solution $\boldsymbol{u}$ to problem (2.1) 2.4 can also be written out as the convolution on $\Gamma$

$$
\int_{\Gamma} \boldsymbol{H}(\boldsymbol{x}, \boldsymbol{y}, \boldsymbol{n}) \boldsymbol{g}(\boldsymbol{y}) d \sigma(\boldsymbol{y})
$$

where $\boldsymbol{H}$ is the Green's tensor associated to the system (2.12.5), and $\boldsymbol{n}$ is the normal to $\Gamma$. The practical determination of this adequate half space Green's tensor $\boldsymbol{H}$ was first studied in [14] and later, more rigorously, in [18]. In particular, $\boldsymbol{H}$ satisfies the decay conditions

$$
\boldsymbol{H}(\boldsymbol{x}, \boldsymbol{y}, \boldsymbol{n})=O\left(|\boldsymbol{x}|^{-2}\right), \nabla_{x} \boldsymbol{H}(\boldsymbol{x}, \boldsymbol{y}, \boldsymbol{n})=O\left(|\boldsymbol{x}|^{-3}\right), \quad|\boldsymbol{x}| \rightarrow \infty,
$$

uniformly in $\boldsymbol{y}$ and in $\boldsymbol{n}$, as long as $\boldsymbol{y}$ remains in a bounded subset of $\mathbb{R}^{3-}$. Due to formula (2.6) we can define a continuous mapping $\mathcal{M}$ from tangential fields $\boldsymbol{g}$ in $H_{0}^{1}(\Gamma)^{2}$ to surface displacement fields $\boldsymbol{u}\left(x_{1}, x_{2}, 0\right)$ in $L^{2}(V)$ where $\boldsymbol{u}$ and $\boldsymbol{g}$ are related by (2.1) 2.5). Theorem 2.2 asserts that this mapping is injective, so an inverse operator can be defined. It is well known, however, that such an operator $\mathcal{M}$ is compact, therefore its inverse is unbounded. It is thus clear that any stable numerical method for reconstructing $\boldsymbol{g}$ from $\boldsymbol{u}\left(x_{1}, x_{2}, 0\right)$ will have to use some regularization process. Our goal in this paper is to analyze the stability properties of the fault inverse problem with regard to the plane containing $\Gamma$, first in section 3 as the slip on the fault is fixed, and then in section 4 in the case of unknown slips.

\section{Lipschitz stability of the fault geometry for a fixed slip}

\subsection{Preliminary results}

The formula for the Green tensor $\boldsymbol{H}(\boldsymbol{x}, \boldsymbol{y}, \boldsymbol{n})$ (2.6) is given in [14, 18]. Here we only give an explicit formula for its free space analog $\boldsymbol{G}(\boldsymbol{x}, \boldsymbol{y}, \boldsymbol{n})$ and we will use the fact that the difference $\boldsymbol{H}(\boldsymbol{x}, \boldsymbol{y}, \boldsymbol{n})-\boldsymbol{G}(\boldsymbol{x}, \boldsymbol{y}, \boldsymbol{n})$ is a smooth function for $(\boldsymbol{x}, \boldsymbol{y})$ in $\mathbb{R}^{3-} \times \mathbb{R}^{3-}$. Recall the well known formula for Kelvin's Green's tensor

$$
\boldsymbol{K}_{i j}(\boldsymbol{x}, \boldsymbol{y})=\frac{1}{8 \pi \mu(\lambda+2 \mu)}\left((\lambda+\mu) \partial_{x_{i}} r \partial_{x_{j}} r+(\lambda+3 \mu) \delta_{i j}\right) \frac{1}{r},
$$

where $r=|\boldsymbol{x}-\boldsymbol{y}|$. Now if $\boldsymbol{v}$ is any fixed vector, define

$$
\boldsymbol{G}(\boldsymbol{x}, \boldsymbol{y}, \boldsymbol{v})=\left(T_{\boldsymbol{v}(y)} \boldsymbol{K}(\boldsymbol{x}, \boldsymbol{y})\right)^{T} .
$$

We will need the following formulas for the jumps across $\Gamma$ of vector fields defined by surface convolution of densities against $\boldsymbol{G}$. 
Lemma 3.1 Let $\Gamma$ be an open surface in $\mathbb{R}^{3}$ included in the plane $x_{3}=0$. Let $\boldsymbol{g}$ be a three dimensional vector field on $\Gamma$ with regularity $C_{c}^{\infty}(\Gamma)$. Then the following jump formulas across $\Gamma$ hold

$$
\begin{array}{r}
{\left[\int_{\Gamma} \boldsymbol{G}\left(\boldsymbol{x}, \boldsymbol{y}, \boldsymbol{e}_{3}\right) \boldsymbol{g}(\boldsymbol{y}) d y_{1} d y_{2}\right]=\boldsymbol{g}(\boldsymbol{x}),} \\
{\left[\int_{\Gamma}\left(\partial_{y_{1}} \boldsymbol{G}\right)\left(\boldsymbol{x}, \boldsymbol{y}, \boldsymbol{e}_{3}\right) \boldsymbol{g}(\boldsymbol{y}) d y_{1} d y_{2}\right]=-\partial_{x_{1}} \boldsymbol{g}(\boldsymbol{x}),} \\
{\left[\int_{\Gamma} \boldsymbol{G}\left(\boldsymbol{x}, \boldsymbol{y}, \boldsymbol{e}_{1}\right) \boldsymbol{g}(\boldsymbol{y}) d y_{1} d y_{2}\right]=\frac{\lambda}{\lambda+2 \mu} g_{1}(\boldsymbol{x}) \boldsymbol{e}_{3}+g_{3}(\boldsymbol{x}) \boldsymbol{e}_{1},} \\
{\left[\int_{\Gamma}\left(\partial_{y_{3}} \boldsymbol{G}\right)\left(\boldsymbol{x}, \boldsymbol{y}, \boldsymbol{e}_{3}\right) \boldsymbol{g}(\boldsymbol{y}) d y_{1} d y_{2}\right]=\frac{\lambda}{\lambda+2 \mu}\left(d i{ }_{\Gamma} \boldsymbol{g}_{\Gamma}\right)(\boldsymbol{x}) \boldsymbol{e}_{3}+\nabla_{\Gamma} g_{3}(\boldsymbol{x}),} \\
{\left[\partial_{x_{3}} \int_{\Gamma} \boldsymbol{G}\left(\boldsymbol{x}, \boldsymbol{y}, \boldsymbol{e}_{3}\right) \boldsymbol{g}(\boldsymbol{y}) d y_{1} d y_{2}\right]=-\frac{\lambda}{\lambda+2 \mu}\left(d i{ }_{\Gamma} \boldsymbol{g}_{\Gamma}\right)(\boldsymbol{x}) \boldsymbol{e}_{3}-\nabla_{\Gamma} g_{3}(\boldsymbol{x}),}
\end{array}
$$

where $\boldsymbol{g}=\left(g_{1}, g_{2}, g_{3}\right), \boldsymbol{g}_{\Gamma}=\left(g_{1}, g_{2}, 0\right)$, div ${ }_{\Gamma} \boldsymbol{g}_{\Gamma}=\partial_{1} g_{1}+\partial_{2} g_{2}$, and $\nabla_{\Gamma}\left(\boldsymbol{g} \cdot \boldsymbol{e}_{3}\right)=\left(\partial_{1} g_{3}, \partial_{2} g_{3}, 0\right)$. For the normal derivative of (3.6) we have the jump formula

$$
\begin{array}{r}
{\left[\partial_{x_{3}} \int_{\Gamma}\left(\partial_{y_{3}} \boldsymbol{G}\right)\left(\boldsymbol{x}, \boldsymbol{y}, \boldsymbol{e}_{3}\right) \boldsymbol{g}(\boldsymbol{y}) d y_{1} d y_{2}\right]=} \\
+\left(\frac{3 \lambda+4 \mu}{\lambda+2 \mu} \partial_{1}^{2} g_{1}+\partial_{2}^{2} g_{1}+2 \frac{\lambda+\mu}{\lambda+2 \mu} \partial_{1} \partial_{2} g_{2}\right) \boldsymbol{e}_{1} \\
+\left(\frac{3 \lambda+4 \mu}{\lambda+2 \mu} \partial_{2}^{2} g_{2}+\partial_{1}^{2} g_{2}+2 \frac{\lambda+\mu}{\lambda+2 \mu} \partial_{1} \partial_{2} g_{1}\right) \boldsymbol{e}_{2} \\
-\frac{\lambda}{\lambda+2 \mu}\left(\partial_{1}^{2}+\partial_{2}^{2}\right) g_{3} \boldsymbol{e}_{3}
\end{array}
$$

Finally, we give a jump formula for the normal derivative of (3.5)

$$
\begin{aligned}
{\left[\partial_{x_{3}} \int_{\Gamma} \boldsymbol{G}\left(\boldsymbol{x}, \boldsymbol{y}, \boldsymbol{e}_{1}\right) \boldsymbol{g}(\boldsymbol{y}) d y_{1} d y_{2}\right]=} & \left(\frac{3 \lambda+4 \mu}{\lambda+2 \mu} \partial_{1} g_{1}+\partial_{2} g_{2}\right) \boldsymbol{e}_{1}+\left(\frac{\lambda}{\lambda+2 \mu} \partial_{2} g_{1}+\partial_{1} g_{2}\right) \boldsymbol{e}_{2} \\
& -\frac{\lambda}{\lambda+2 \mu} \partial_{1} g_{3} \boldsymbol{e}_{3} .
\end{aligned}
$$

Proof: Formula (3.3) is well known, however, formulas (3.4 3.9) are not readily found in the literature. It is therefore worth providing a proof, which can be found in the Appendix.

Lemma 3.2 Let $\boldsymbol{g}$ be be a tangential vector field on $\Gamma$ with regularity $H_{0}^{1}(\Gamma)$. Then the jump formulas (3.3) and (3.5) still hold in the $H_{0}^{1}(\Gamma)$ norm, while the jump formulas 3.4. 3.6. (3.7. 3.9) hold as continuous linear operations from $H_{0}^{1}(\Gamma)$ to $L^{2}(\Gamma)$. The jump formula (3.8) holds as a continuous linear operator from $H_{0}^{1}(\Gamma)$ to $H^{-1}(\Gamma)$.

Proof: This is clear since $C_{c}^{\infty}(\Gamma)$ is dense in $H_{0}^{1}(\Gamma)$.

Lemma 3.3 Let $\Omega$ be an open bounded subset in $\mathbb{R}^{2}$. Let $f$ be in $C^{\infty}\left(\mathbb{R}^{2}\right)$ such that $f^{-1}(\{0\}) \cap \Omega$ has zero measure. Let $\boldsymbol{\tau}$ be a non-zero vector in $\mathbb{R}^{2}$. Assume that $u$ is in $H_{0}^{1}(\Omega)$ and satisfies in $\Omega$ the partial differential equation

$$
\partial_{\boldsymbol{\tau}}(f u)+\alpha u=0,
$$

where $\alpha$ is a constant in $\mathbb{R}$. Then $u$ is zero. 
Proof: We first assume that $\alpha \neq 0$. We note that for any function $g$ in $C_{c}^{\infty}(\Omega)$ the divergence theorem implies that

$$
0=\int_{\Omega} \operatorname{div}(g \tau)=\int_{\Omega} \nabla g \cdot \tau
$$

which can be extended by density to any $g$ in $H_{0}^{1}(\Omega)$. Let $f_{n}$ be a sequence in $C^{\infty}(\Omega)$ which converges to $f^{+}$in the $H^{1}$ norm. By formula (3.11),

$$
0=\int_{\Omega} \nabla\left(f_{n} f u^{2}\right) \cdot \boldsymbol{\tau}=\int_{\Omega} f_{n} u \nabla(f u) \cdot \boldsymbol{\tau}+f u \nabla\left(f_{n} u\right) \cdot \boldsymbol{\tau}
$$

Next we want to prove that

$$
\lim _{n \rightarrow \infty} \int_{\Omega} f_{n} u \nabla(f u)=\lim _{n \rightarrow \infty} \int_{\Omega} f u \nabla\left(f_{n} u\right)=\int_{\Omega} f^{+} u \nabla(f u) .
$$

The first limit in (3.13) is clear. We observe that

$$
f_{n} u \nabla(f u)-f u \nabla\left(f_{n} u\right)=u^{2}\left(f \nabla f_{n}-f_{n} \nabla f\right)
$$

Since $\Omega$ is two dimensional and $u$ is $H_{0}^{1}(\Omega)$, we can assert by the Sobolev embeddings that $u^{2}$ is in $L^{2}(\Omega) . f \nabla f_{n}-f_{n} \nabla f$ converges to $f \nabla f^{+}-f^{+} \nabla f$ in $L^{2}(\Omega)$, which is zero, so the second limit in (3.13) is proved. Going back to (3.11), we have now shown,

$$
\int_{\Omega} f^{+} u \nabla(f u) \cdot \tau=0
$$

We now multiply equation (3.10) by $f^{+} u$, we integrate over $\Omega$, and we use that $\alpha$ is non-zero to find that $\int_{\Omega} f^{+} u^{2}$ is zero. Similarly, $\int_{\Omega} f^{-} u^{2}$ is zero. As $f^{-1}(\{0\}) \cap \Omega$ has measure zero, this shows that $u$ is zero.

We now consider the case where $\alpha$ is zero. After a linear change of variables, we may assume that $\boldsymbol{\tau}$ is the base vector $\boldsymbol{e}_{1}$. Let $A$ be a constant such that $\Omega$ is included in the box $-A \leq x_{1}, x_{2} \leq A$. We first note that for any function $g$ in $C_{c}^{1}((-A, A) \times(-A, A))$ for any $-A \leq x_{1}, x_{2} \leq A$,

$$
\left|g\left(x_{1}, x_{2}\right)\right| \leq\left(\int_{-A}^{A}\left|\partial_{x_{1}} g\left(x_{1}, x_{2}\right)\right|^{2} d x_{1}\right)^{1 / 2}(2 A)^{1 / 2}
$$

thus

$$
\int_{-A}^{A} \int_{-A}^{A}\left|g\left(x_{1}, x_{2}\right)\right|^{2} d x_{1} d x_{2} \leq 4 A^{2} \int_{-A}^{A} \int_{-A}^{A}\left|\partial_{x_{1}} g\left(x_{1}, x_{2}\right)\right|^{2} d x_{1} d x_{2}
$$

and this estimate can be extended to all $g$ in $H_{0}^{1}((-A, A) \times(-A, A))$. It thus follows that $f u$ in zero in $(-A, A) \times(-A, A)$, thus $u$ is zero in $\Omega$.

\subsection{Lipschitz stability theorem for a fixed slip}

Let $R$ be a closed rectangle in the plane $x_{3}=0$. Let $B$ be a set of triplets $(a, b, d)$ such that the set

$$
\Gamma_{a, b, d}=\left\{\left(x_{1}, x_{2}, a x_{1}+b x_{2}+d\right):\left(x_{1}, x_{2}\right) \in R\right\}
$$


is included in the half-space $x_{3}<0$. When appropriate, we will use the short hand notation $m=(a, b, d)$. We assume that $B$ is a closed and bounded subset of $\mathbb{R}^{3}$. It follows that that

the distance between $\Gamma_{m}$ and the plane $x_{3}=0$ is bounded below by the same positive constant for all $m$ in $B$.

We set $\boldsymbol{n}=(-a,-b, 1) / \sqrt{1+a^{2}+b^{2}}$ to be the normal vector on $\Gamma_{m}$ and $\sigma=\sqrt{1+a^{2}+b^{2}}$ the surface element. Let $H_{0}^{1}(R)^{2}$ be the space of vector fields $\boldsymbol{g}=\left(g_{1}, g_{2}\right)$ on $R$ with $H_{0}^{1}$ regularity. Define $\boldsymbol{g}_{m}$ the tangential vector field on $\Gamma_{m}, \boldsymbol{g}_{m}=\left(g_{1}, g_{2}, a g_{1}+b g_{2}\right)$. Define the operator

$$
\begin{aligned}
A_{m}: & H_{0}^{1}(R)^{2} \rightarrow L^{2}(V)^{3} \\
& \boldsymbol{g} \rightarrow \int_{R} \boldsymbol{H}\left(\boldsymbol{x}, y_{1}, y_{2}, a y_{1}+b y_{2}+d, \boldsymbol{n}\right) \boldsymbol{g}_{m}\left(y_{1}, y_{2}\right) \sigma d y_{1} d y_{2} .
\end{aligned}
$$

It is clear that $A_{m}$ is linear, continuous, and compact. Note that due to Theorem $2.2, A_{m}$ is injective. In the remainder of this section we fix a non zero $\boldsymbol{h}$ in $H_{0}^{1}(R)$, and we define a non-linear function

$$
\begin{array}{r}
\phi: B \rightarrow L^{2}(V)^{3} \\
\phi(m)=\int_{R} \boldsymbol{H}\left(\boldsymbol{x}, y_{1}, y_{2}, a y_{1}+b y_{2}+d, \boldsymbol{n}\right) \boldsymbol{h}_{m}\left(y_{1}, y_{2}\right) \sigma d y_{1} d y_{2},
\end{array}
$$

where $m=(a, b, d)$. Due to the regularity of the Green's tensor $\boldsymbol{H}(\boldsymbol{x}, \boldsymbol{y}, \boldsymbol{n})$, it is clear that $\phi$ is real analytic in $m$. Now due to Theorem 2.2, $\phi$ is injective. We now want to prove that the inverse of $\phi$ defined on $\phi(B)$ and valued in $B$ is Lipschitz continuous. This will be achieved by showing that we can apply the inverse function Theorem.

Theorem 3.1 Fix a non-zero $\boldsymbol{h}$ in $H_{0}^{1}(R)^{2}$ and define the function $\phi$ from $B$ to $L^{2}(V)^{3}$ by (3.16). Assume that:

(i). $B$ does not contain any triplet in the form $(0,0, d)$, in other words no horizontal profiles are allowed for the faults.

or

(ii). $\boldsymbol{h}$ is one-directional.

or

(iii). $\boldsymbol{h}$ is in $H_{0}^{2}(R)^{2}$.

There is a positive constant $C$ such that

$$
C\left|m-m^{\prime}\right| \leq\left\|\phi(m)-\phi\left(m^{\prime}\right)\right\|_{L^{2}(V)},
$$

for all $m$ and $m^{\prime}$ in $B$.

Proof: Fix $m$ in $B$. Our first task is to evaluate $\nabla \phi(m)$. We first note that $\boldsymbol{n} \sigma$ simplifies to $(-a,-b, 1)$. We recall that $\boldsymbol{H}(\boldsymbol{x}, \boldsymbol{y}, \boldsymbol{n})$ is linear in $\boldsymbol{n}$. By the chain rule, for $\boldsymbol{y}=a y_{1}+b y_{2}+d$,

$$
\begin{aligned}
\frac{\partial}{\partial a} \boldsymbol{H}(\boldsymbol{x}, \boldsymbol{y}, \boldsymbol{n} \sigma) & =\frac{\partial y_{3}}{\partial a}\left(\partial_{y_{3}} \boldsymbol{H}\right)(\boldsymbol{x}, \boldsymbol{y}, \boldsymbol{n} \sigma)-\boldsymbol{H}\left(\boldsymbol{x}, \boldsymbol{y}, \boldsymbol{e}_{1}\right) \\
& =y_{1}\left(\partial_{y_{3}} \boldsymbol{H}\right)(\boldsymbol{x}, \boldsymbol{y}, \boldsymbol{n} \sigma)-\boldsymbol{H}\left(\boldsymbol{x}, \boldsymbol{y}, \boldsymbol{e}_{1}\right) .
\end{aligned}
$$

Similarly,

$$
\frac{\partial}{\partial b} \boldsymbol{H}(\boldsymbol{x}, \boldsymbol{y}, \boldsymbol{n})=y_{2}\left(\partial_{y_{3}} \boldsymbol{H}\right)(\boldsymbol{x}, \boldsymbol{y}, \boldsymbol{n} \sigma)-\boldsymbol{H}\left(\boldsymbol{x}, \boldsymbol{y}, \boldsymbol{e}_{2}\right)
$$


and

$$
\frac{\partial}{\partial d} \boldsymbol{H}(\boldsymbol{x}, \boldsymbol{y}, \boldsymbol{n})=\left(\partial_{y_{3}} \boldsymbol{H}\right)(\boldsymbol{x}, \boldsymbol{y}, \boldsymbol{n} \sigma) .
$$

Arguing by contradiction, assume that for some $m$ in $B, \nabla \phi(m)$ does not have full rank. Then there is a non-zero vector $\left(\gamma_{1}, \gamma_{2}, \gamma_{3}\right)$ in $\mathbb{R}^{3}$ such that

$$
\gamma_{1} \frac{\partial}{\partial a} \phi(m)+\gamma_{2} \frac{\partial}{\partial b} \phi(m)+\gamma_{3} \frac{\partial}{\partial d} \phi(m)=0 .
$$

Set $f\left(y_{1}, y_{2}\right)=\gamma_{1} y_{1}+\gamma_{2} y_{2}+\gamma_{3}$. We note that $\left(\gamma_{1} \frac{\partial}{\partial a}+\gamma_{2} \frac{\partial}{\partial b}+\gamma_{3} \frac{\partial}{\partial d}\right) \boldsymbol{h}_{m}=\nabla f \cdot \boldsymbol{h}_{m} \boldsymbol{e}_{3}$. Relation (3.19) can be expressed as

$$
\begin{array}{r}
\int_{R} \boldsymbol{H}\left(\boldsymbol{x}, y_{1}, y_{2}, a y_{1}+b y_{2}+d, \boldsymbol{n}\right) \nabla f \cdot \boldsymbol{h}_{m}\left(y_{1}, y_{2}\right) \boldsymbol{e}_{3} \sigma d y_{1} d y_{2} \\
\int_{R}\left(\partial_{y_{3}} \boldsymbol{H}\right)\left(\boldsymbol{x}, y_{1}, y_{2}, a y_{1}+b y_{2}+d, \boldsymbol{n}\right) \boldsymbol{h}_{m}\left(y_{1}, y_{2}\right) f\left(y_{1}, y_{2}\right) \sigma d y_{1} d y_{2} \\
-\int_{R} \boldsymbol{H}\left(\boldsymbol{x}, y_{1}, y_{2}, a y_{1}+b y_{2}+d, \nabla f\right) \boldsymbol{h}_{m}\left(y_{1}, y_{2}\right) d y_{1} d y_{2}=0,
\end{array}
$$

for all $\boldsymbol{x}$ in $V$. Set $\boldsymbol{w}(\boldsymbol{x})$ to be the left hand side of (3.20) where $\boldsymbol{x}$ has been extended to $\mathbb{R}^{3-} \backslash \Gamma_{m}$. We now proceed to prove that $\boldsymbol{w}$ is zero in $\mathbb{R}^{3-} \backslash \Gamma_{m}$. First, it is clear that $\boldsymbol{w}$ satisfies the elasticity equations in $\mathbb{R}^{3-} \backslash \Gamma_{m}$ since the scalar differential operators $\partial_{y_{3}}$ and $\partial_{x_{j}}$ commute. Next, due to (3.20), $\boldsymbol{w}$ is zero on $V$. By construction of Green's tensor $\boldsymbol{H}$, for any $\boldsymbol{x}$ on the plane $x_{3}=0$, any $\boldsymbol{y}$ in $\mathbb{R}^{3-}$, and any fixed vector $\boldsymbol{p}$ in $\mathbb{R}^{3}$,

$$
T_{\boldsymbol{e}_{3}}(\boldsymbol{x}) \boldsymbol{H}(\boldsymbol{x}, \boldsymbol{y}, \boldsymbol{p})=0 .
$$

We can thus take a $\partial_{y_{3}}$ derivative and commute the matrix differential operator $T_{\boldsymbol{e}_{3}}(\boldsymbol{x})$ with the scalar differential operator $\partial_{y_{3}}$ to obtain

$$
T_{\boldsymbol{e}_{3}}(\boldsymbol{x}) \partial_{y_{3}} \boldsymbol{H}(\boldsymbol{x}, \boldsymbol{y}, \boldsymbol{p})=0 .
$$

It follows that $T_{\boldsymbol{e}_{3}} \boldsymbol{w}$ is also zero in $V$ and a Cauchy Kowaleski type argument as in the proof of Theorem 2.2. which was given in $\underline{22}$, shows that $\boldsymbol{w}$ must be zero everywhere in $\mathbb{R}^{3-} \backslash \Gamma_{m}$. In particular the jump of $\boldsymbol{w}$ across $\Gamma_{m}$ must also be zero. Recall the definition of $\boldsymbol{G}$ given by (3.2). We note that for any vector $\boldsymbol{v}$ in $\mathbb{R}^{3}, \boldsymbol{H}(\boldsymbol{x}, \boldsymbol{y}, \boldsymbol{v})-\boldsymbol{G}(\boldsymbol{x}, \boldsymbol{y}, \boldsymbol{v})$ is smooth for all $\boldsymbol{x}$ and $\boldsymbol{y}$ in $\mathbb{R}^{3-}$, see 18 . Therefore, the jump across $\Gamma_{m}$ of

$$
\begin{array}{r}
\int_{R} \boldsymbol{G}\left(\boldsymbol{x}, y_{1}, y_{2}, a y_{1}+b y_{2}+d, \boldsymbol{n}\right) \nabla f \cdot \boldsymbol{h}\left(y_{1}, y_{2}\right) \boldsymbol{e}_{3} \sigma d y_{1} d y_{2} \\
\int_{R}\left(\partial_{y_{3}} \boldsymbol{G}\right)\left(\boldsymbol{x}, y_{1}, y_{2}, a y_{1}+b y_{2}+d, \boldsymbol{n}\right) \boldsymbol{h}_{m}\left(y_{1}, y_{2}\right) f\left(y_{1}, y_{2}\right) \sigma d y_{1} d y_{2} \\
-\int_{R} \boldsymbol{G}\left(\boldsymbol{x}, y_{1}, y_{2}, a y_{1}+b y_{2}+d, \nabla f\right) \boldsymbol{h}_{m}\left(y_{1}, y_{2}\right) d y_{1} d y_{2},
\end{array}
$$

is also zero. Let us write

$$
\boldsymbol{e}_{3}=\alpha \boldsymbol{n}+\boldsymbol{\tau}
$$


where $\tau$ is parallel to $\Gamma_{m}$. We now use the fact that the free space Green's function is rotation invariant. After a change of coordinates by rotation, we can assume that $\Gamma_{m}$ is horizontal and $\boldsymbol{\tau}=\beta \boldsymbol{e}_{1}$ (for the sake of lighter notations, the new coordinates will be named in the same way as the old coordinates). In the new coordinates we note that $\boldsymbol{h}_{m} \cdot \boldsymbol{e}_{3}=0$, and we simply write $\boldsymbol{h}$ in place of $\boldsymbol{h}_{m}$. The expression (3.21) can be written out as

$$
\begin{array}{r}
\int_{R} \boldsymbol{G}\left(\boldsymbol{x}, y_{1}, y_{2}, \tilde{d}, \boldsymbol{e}_{3}\right)(\nabla \tilde{f} \cdot \boldsymbol{h})\left(\alpha \boldsymbol{e}_{3}+\beta \boldsymbol{e}_{1}\right) \sigma d y_{1} d y_{2} \\
+\alpha \int_{R} \partial_{y_{3}} \boldsymbol{G}\left(\boldsymbol{x}, y_{1}, y_{2}, \tilde{d}, \boldsymbol{e}_{3}\right) \boldsymbol{h} \tilde{f} \sigma d y_{1} d y_{2} \\
+\beta \int_{R} \partial_{y_{1}} \boldsymbol{G}\left(\boldsymbol{x}, y_{1}, y_{2}, \tilde{d}, \boldsymbol{e}_{3}\right) \boldsymbol{h} \tilde{f} \sigma d y_{1} d y_{2} \\
-\int_{R} \boldsymbol{G}\left(\boldsymbol{x}, y_{1}, y_{2}, \tilde{d}, \nabla \tilde{f}\right) \boldsymbol{h} d y_{1} d y_{2},
\end{array}
$$

where $\tilde{f}$ is a non -zero affine function. This must also have a zero jump across $R+\tilde{d}$. We now proceed to write down the expression for that jump thanks Lemma 3.2 and formulas (3.3.3.6) to find

$$
\begin{array}{r}
(\nabla \tilde{f} \cdot \boldsymbol{h})\left(\alpha \boldsymbol{e}_{3}+\beta \boldsymbol{e}_{1}\right) \sigma \\
+\alpha \sigma \frac{\lambda}{\lambda+2 \mu}(\operatorname{div}(\tilde{f} \boldsymbol{h})) \boldsymbol{e}_{3} \\
-\beta \sigma \partial_{1}(\tilde{f} \boldsymbol{h}) \\
-\partial_{3} \tilde{f} \boldsymbol{h} \\
-\frac{\lambda}{\lambda+2 \mu} \boldsymbol{h} \cdot \nabla \tilde{f} \boldsymbol{e}_{3}=0 .
\end{array}
$$

As $\sigma \alpha=1$, this simplifies along $\boldsymbol{e}_{3}$ to

$$
\frac{\lambda}{\lambda+2 \mu} \tilde{f} \operatorname{div} \boldsymbol{h}+\nabla \tilde{f} \cdot \boldsymbol{h}=0 .
$$

The remaining terms lead to the equation

$$
\beta \sigma(\nabla \tilde{f} \cdot \boldsymbol{h}) \boldsymbol{e}_{1}-\beta \sigma \partial_{1}(\tilde{f} \boldsymbol{h})-\partial_{3} \tilde{f} \boldsymbol{h}=0,
$$

that is, to the system

$$
\begin{aligned}
-\beta \sigma \partial_{1}\left(\tilde{f} h_{2}\right)-\partial_{3} \tilde{f} h_{2} & =0 \\
-\beta \sigma \partial_{1}\left(\tilde{f} h_{1}\right)+\beta \sigma\left(\partial_{1} \tilde{f} h_{1}+\partial_{2} \tilde{f} h_{2}\right)-\partial_{3} \tilde{f} h_{1} & =0
\end{aligned}
$$

Assume that condition (i) in the statement of Theorem 3.1 holds. Then $\Gamma_{m}$ is not horizontal, thus $\beta \neq 0$. Note that $\nabla \tilde{f}$ is a constant vector. Then we can use the first line of equation (3.23) in conjunction to Lemma 3.3 to find that $h_{2}=0$. Then due to the second line of (3.23) and Lemma $3.3 h_{1}=0$. Thus we showed that $\boldsymbol{h}$ is zero in $H_{0}^{1}(R)^{2}$ : contradiction. If condition (ii) in the statement of Theorem 3.1 holds, we set $\boldsymbol{h}=u V$, where $u$ is a scalar function and $V$ is a fixed vector and equation (3.22) simplifies to

$$
\frac{\lambda}{\lambda+2 \mu} V \cdot \nabla(\tilde{f} u)+\frac{2 \mu}{\lambda+2 \mu}(V \cdot \nabla \tilde{f}) u,
$$


so equation (3.22) in conjunction to Lemma 3.3 can be used to show that $u$ is zero.

Now, assume that $\Gamma_{m}$ is horizontal and that condition (iii) holds. In that case $\beta=0$ and equation (3.23) is void. We also note that here $\alpha=\sigma=1$ and that equation (3.22) is still valid. We use that the jump of the $\partial_{x_{3}}$ derivative across $\Gamma_{m}$ of

$$
\begin{array}{r}
\int_{\Gamma_{m}} \boldsymbol{G}\left(\boldsymbol{x}, y_{1}, y_{2}, d, \boldsymbol{e}_{3}\right)(\nabla f \cdot \boldsymbol{h}) \boldsymbol{e}_{3} d y_{1} d y_{2} \\
+\int_{\Gamma_{m}} \partial_{y_{3}} \boldsymbol{G}\left(\boldsymbol{x}, y_{1}, y_{2}, d, \boldsymbol{e}_{3}\right) \boldsymbol{h} f d y_{1} d y_{2} \\
\quad-\int_{\Gamma_{m}} \boldsymbol{G}\left(\boldsymbol{x}, y_{1}, y_{2}, d, \nabla f\right) \boldsymbol{h} d y_{1} d y_{2}
\end{array}
$$

is zero. To complete the proof we apply a change of coordinates by rotation about $\boldsymbol{e}_{3}$ such that $\nabla f$ becomes parallel to $\boldsymbol{e}_{1}$ in the new coordinates. By homogeneity, we can then assume that $f\left(x_{1}, x_{2}\right)=x_{1}+\gamma_{3}$.

We now apply formula (3.7] 3.9 ) to the (zero) $\partial_{x_{3}}$ jump of (3.24) to obtain the following equation in the direction of $\boldsymbol{e}_{1}$

$$
\begin{array}{r}
-\partial_{1}\left(\partial_{1} f h_{1}\right)+\frac{3 \lambda+4 \mu}{\lambda+2 \mu} \partial_{1}^{2}\left(f h_{1}\right)+\partial_{2}^{2}\left(f h_{1}\right)+2 \frac{\lambda+\mu}{\lambda+2 \mu} \partial_{1} \partial_{2}\left(f h_{2}\right) \\
-\partial_{1} f\left(\frac{3 \lambda+4 \mu}{\lambda+2 \mu} \partial_{1} h_{1}+\partial_{2} h_{2}\right)=0 .
\end{array}
$$

We then eliminate $h_{2}$ in (3.25). This is done by using (3.22) and observing that as $\partial_{1} f=1$,

$$
\partial_{1} \partial_{2}\left(f h_{2}\right)=-\partial_{1}^{2}\left(f h_{1}\right)-2 \frac{\mu}{\lambda} \partial_{1} h_{1}
$$

so (3.25) reduces to, as $1+4 \frac{\mu}{\lambda} \frac{\lambda+\mu}{\lambda+2 \mu}+\frac{3 \lambda+4 \mu}{\lambda+2 \mu}=4+2 \frac{\mu}{\lambda}$,

$$
\partial_{1}^{2}\left(f h_{1}\right)+\partial_{2}^{2}\left(f h_{1}\right)+\partial_{1} h_{1}\left(-4-2 \frac{\mu}{\lambda}\right)-\partial_{2} h_{2}=0
$$

We multiply by $f$, use again (3.22) and simplify to obtain

$$
f^{2} \Delta h_{1}+f \partial_{1} h_{1}\left(-3-2 \frac{\mu}{\lambda}\right)+\left(1+2 \frac{\mu}{\lambda}\right) f h_{1}=0 .
$$

Note that this not an elliptic PDE as $f$ may be equal to zero in $\Gamma$. To show that $h_{1}$ is zero, fix $\epsilon>0$, let $\Gamma^{+}=\{x \in \Gamma: f(x)>\epsilon\}$ and $\Gamma^{-}=\{x \in \Gamma: f(x)<-\epsilon\}$. As $\boldsymbol{h}$ is in $H_{0}^{2}(\Gamma)$, since $\Gamma^{+}$is the intersection of $\Gamma$ and a half plane, if it is non empty, the Cauchy Kowaleski Theorem can be applied to (3.27) to claim that $h_{1}$ is zero in $\Gamma^{+}$. We carry out the same argument on $\Gamma^{-}$. Finally we let $\epsilon$ tend to zero: this proves that $h_{1}$ is zero in $\Gamma$. From there we claim that $h_{2}$ is also zero by recalling (3.22) and applying Lemma 3.3

We have thus proved that for all $m$ in $B, \nabla \phi(m)$ has full rank. We now include the set $B$ in a subset $B^{\prime}$ of $\mathbb{R}^{3}$ such that $B^{\prime}$ is open and property (3.14) still holds for $B^{\prime}$. As for every $m$ in $B^{\prime}, \nabla \phi(m)$ has full rank, by the inverse function theorem $\phi$ defines a $C^{1}$ diffeomorphism from an open neighborhood $U_{m}$ to its image by $\phi$ on $L^{2}(V)$. Thus, there is a positive constant $C_{m}$ such that for all $m^{\prime}$ and $m^{\prime \prime}$ in $U_{m}$,

$$
C_{m}\left|m^{\prime}-m^{\prime \prime}\right| \leq\left\|\phi\left(m^{\prime}\right)-\phi\left(m^{\prime \prime}\right)\right\|_{L^{2}(V)} .
$$


Arguing by contradiction, assume that estimate (3.18) fails to be true. Then there are two sequences $m_{n}^{\prime}$ and $m_{n}^{\prime \prime}$ in $B$ such that $m_{n}^{\prime} \neq m_{n}^{\prime \prime}$ and $\frac{\left\|\phi\left(m_{n}^{\prime}\right)-\phi\left(m_{n}^{\prime \prime}\right)\right\|_{L^{2}(V)}}{\left|m_{n}^{\prime}-m_{n}^{\prime \prime}\right|}$ tends to zero. As $B$ is compact, we may assume after extracting subsequences that $m_{n}^{\prime}$ converges to $\tilde{m}$ and $m_{n}^{\prime \prime}$ converges to $\tilde{\tilde{m}}$. Since $\phi$ is continuous and injective we must have $\tilde{m}=\tilde{\tilde{m}}$. But for all $n$ large enough $m_{n}^{\prime}$ and $m_{n}^{\prime \prime}$ must be in the open neighborhood $U_{\tilde{m}}$ : contradiction.

\section{Second stability theorem: the case of unknown slips}

In applications the slip on $\Gamma$ is unknown, therefore this slip cannot be used to minimize $\left\|\phi(m)-\phi\left(m_{0}\right)\right\|_{L^{2}(V)}$ for $m$ over $B$ as in (3.18) to find the geometry $m_{0}$. Instead, one has to minimize $\left\|A_{m} \boldsymbol{h}-A_{m_{0}} \boldsymbol{h}_{0}\right\|_{L^{2}(V)}$ over all geometries $m$ and all slips $\boldsymbol{h}$. The unique minimum is zero and only achieved for $m=m_{0}$ and $\boldsymbol{h}=\boldsymbol{h}_{0}$ according to Theorem 2.2. To obtain Lipschitz stability in $\left|m-m_{0}\right|$ we need to add an additional assumption on $\boldsymbol{h}_{0}$. A possible additional assumption is to require that $\boldsymbol{h}_{0}$ be one directional. Physically, this means that the slip on the fault $\Gamma$ occurs in only one direction. Interestingly, this condition already appeared in another theoretical study of destabilization modes of faults, [20, as discussed in section 2.2

Recall the definition (3.15) of operator $A_{m}$. We will need the following lemma.

Lemma 4.1 Let $P_{m}$ be the orthogonal projection onto $\overline{R\left(A_{m}\right)}$ in $L^{2}(V)$. Fix $m_{0}$ in $B$. Then there is a constant $C$ such that

$$
\left\|P_{m}-P_{m_{0}}\right\| \leq C\left|m-m_{0}\right|
$$

for all $m$ in $B$.

Proof: We first note that the closure $\overline{R\left(A_{m}\right)}$ of the range of $A_{m}$ in $L^{2}(V)$ is equal to $\overline{R\left(A_{m} A_{m}^{*}\right)}$ : this is true because the nullspace $N\left(A_{m}^{*}\right)$ is equal to $N\left(A_{m} A_{m}^{*}\right)$ and we can then take the orthogonals of each of this subspace. If $m$ tends to $m_{0}$, it is clear $A_{m} A_{m}^{*}$ is norm convergent to $A_{m_{0}} A_{m_{0}}^{*}$ and that $\left\|A_{m} A_{m}^{*}-A_{m_{0}} A_{m_{0}}^{*}\right\|=O\left(\left|m-m_{0}\right|\right)$. Let $\mathcal{C}$ be the circle in the complex plane centered at the origin with radius $\left\|A_{m_{0}} A_{m_{0}}^{*}\right\|+1$. The orthogonal projection on the image of $A_{m} A_{m}^{*}$ can be represented by the contour integral as follows, see [12],

$$
P_{m}=\frac{1}{2 i \pi} \int_{\mathcal{C}}\left(\zeta I-A_{m} A_{m}^{*}\right)^{-1} d \zeta
$$

for all $m$ large enough, and where $I$ is the identity operator in $L^{2}(V)$. This leads to (4.1).

Theorem 4.1 Fix a non-zero $\boldsymbol{h}_{0}$ in $H_{0}^{1}(R)$ and $m_{0}$ in $B$. Assume that $\boldsymbol{h}_{0}$ satisfies one of the two following additional assumptions:

(i). $\boldsymbol{h}_{0}$ is one-directional, that is, $\boldsymbol{h}_{0}$ is parallel to a fixed tangential vector.

(ii). $\boldsymbol{h}_{0}$ is the gradient of a function $\varphi$ in $H^{2}(\Gamma)$.

Then there exists a positive constant $C$ such that

$$
\inf _{\boldsymbol{h} \in H_{0}^{1}(R)}\left\|A_{m} \boldsymbol{h}-A_{m_{0}} \boldsymbol{h}_{0}\right\|_{L^{2}(V)} \geq C\left|m-m_{0}\right|,
$$

for all $m$ in $B$. 
Proof: Since $I-P_{m}$ is an orthogonal projection,

$$
\left\|A_{m} \boldsymbol{h}-A_{m_{0}} \boldsymbol{h}_{0}\right\|_{L^{2}(V)}^{2} \geq\left\|\left(I-P_{m}\right)\left(A_{m} \boldsymbol{h}-A_{m_{0}} \boldsymbol{h}_{0}\right)\right\|_{L^{2}(V)}^{2} .
$$

Since $P_{m}$ is the orthogonal projection on $\overline{R\left(A_{m}\right)}, P_{m} A_{m} \boldsymbol{h}=A_{m} \boldsymbol{h}$, and we obtain

$$
\left.\left\|A_{m} \boldsymbol{h}-A_{m_{0}} \boldsymbol{h}_{0}\right\|_{L^{2}(V)}^{2} \geq \|\left(I-P_{m}\right) A_{m_{0}} \boldsymbol{h}_{0}\right)\left\|_{L^{2}(V)}^{2}=\right\| P_{m} A_{m_{0}} \boldsymbol{h}_{0}-A_{m_{0}} \boldsymbol{h}_{0} \|_{L^{2}(V)}^{2} .
$$

Arguing by contradiction, assume that there is a sequence $m_{n}$ in $B$ converging to $m_{0}$ such that

$$
\left\|P_{m_{n}} A_{m_{0}} \boldsymbol{h}_{0}-A_{m_{0}} \boldsymbol{h}_{0}\right\|_{L^{2}(V)}=o\left(\left|m_{n}-m_{0}\right|\right) .
$$

It clearly follows that

$$
\left\|\left(I-P_{m_{n}}\right)\left(A_{m_{n}}-A_{m_{0}}\right) \boldsymbol{h}_{0}\right\|_{L^{2}(V)}=o\left(\left|m_{n}-m_{0}\right|\right) .
$$

As

$$
\left\|\left(P_{m_{0}}-P_{m_{n}}\right)\left(A_{m_{n}}-A_{m_{0}}\right) \boldsymbol{h}_{0}\right\|_{L^{2}(V)}=o\left(\left|m_{n}-m_{0}\right|\right),
$$

we may write

$$
\left\|\left(I-P_{m_{0}}\right)\left(A_{m_{n}}-A_{m_{0}}\right) \boldsymbol{h}_{0}\right\|_{L^{2}(V)}=o\left(\left|m_{n}-m_{0}\right|\right) .
$$

Equivalently,

$$
\left(I-P_{m_{0}}\right) \frac{\left(A_{m_{n}}-A_{m_{0}}\right)}{\left|m_{n}-m_{0}\right|} \boldsymbol{h}_{0}=o(1)
$$

As $\frac{m_{n}-m_{0}}{\left|m_{n}-m_{0}\right|}$ is a sequence on the unit sphere of $\mathbb{R}^{3}$, after possibly extracting a subsequence we may assume that it converges to some $\boldsymbol{q}$ with $|\boldsymbol{q}|=1$. Taking the limit as $n \rightarrow \infty$ in (4.4) we find,

$$
\left(I-P_{m_{0}}\right) \partial_{\boldsymbol{q}} A_{m_{0}} \boldsymbol{h}_{0}=0,
$$

thus, there is a $\boldsymbol{g}_{0}$ in $H_{0}^{1}(R)$ such that

$$
\partial_{\boldsymbol{q}} A_{m_{0}} \boldsymbol{h}_{0}=A_{m_{0}} \boldsymbol{g}_{0}
$$

We then set $\boldsymbol{q}=\left(\gamma_{1}, \gamma_{2}, \gamma_{3}\right)$ as in the proof of Theorem 3.1. Given the form (3.15) of the operator $A_{m}$ for $m$ in $B, A_{m_{0}} \boldsymbol{g}_{0}$ can be extended to a vector field on $\mathbb{R}^{3-} \backslash \Gamma_{m_{0}}$ satisfying equations (2.1-2.5) with $\boldsymbol{g}_{0}$ in place of $\boldsymbol{g}$ and $\Gamma_{m_{0}}$ in place of $\Gamma$. In particular, the normal jump of that extended vector field across $\Gamma_{m_{0}}$ is zero. The same argument as in the proof of Theorem 3.1 can then be carried out to show that $\boldsymbol{h}_{0}$ must satisfy, due to (4.5), a partial differential equation on $\Gamma_{m_{0}}$. Due to the $A_{m_{0}} \boldsymbol{g}_{0}$ term on the right hand side this equation will be unhelpful along any direction which is tangential to $\Gamma_{m_{0}}$. However we obtain the same homogeneous equation in the normal direction which we write here for $\boldsymbol{h}_{0}$

$$
\frac{\lambda}{\lambda+2 \mu} f \operatorname{div} \boldsymbol{h}_{0}+\nabla f \cdot \boldsymbol{h}_{0}=0
$$


where this equation was written in a rotated coordinate system such that $\Gamma_{m_{0}}$ is parallel to the new $x_{1}, x_{2}$ plane, $\boldsymbol{h}_{0}$ depends only on the new coordinates $x_{1}, x_{2}$ and $f$ is a non-zero affine function whose coefficients depend linearly on $\gamma_{1}, \gamma_{2}, \gamma_{3}$.

If assumption (i) on $\boldsymbol{h}_{0}$ holds then we can apply lemma 3.3 to claim that $\boldsymbol{h}_{0}$ is zero: contradiction.

If assumption (ii) on $\boldsymbol{h}_{0}$ holds then $\varphi$ satisfies the partial differential equation

$$
\frac{\lambda}{\lambda+2 \mu} f \Delta \varphi+\nabla f \cdot \nabla \varphi=0
$$

Let $\operatorname{sgn}_{0}$ be the sign function defined on $\mathbb{R}$ by: $\operatorname{sgn}_{0}(t)=-1$ if $t<0, \operatorname{sgn}_{0}(0)=0$ and $\operatorname{sgn}_{0}(t)=1$ if $t>0$. Multiplying (4.7) by $\left(1+\frac{2 \mu}{\lambda}\right)|f|^{\frac{2 \mu}{\lambda}} \operatorname{sgn}_{0}(f)$, we obtain

$$
\operatorname{div}\left(|f|^{1+\frac{2 \mu}{\lambda}} \nabla \varphi\right)=0 .
$$

As by assumption $\boldsymbol{h}_{0}=\nabla \varphi$ is in $H_{0}^{1}(\Gamma)$, multiplying by $\varphi$ and applying Green's theorem leads to

$$
\int_{\Gamma}|f|^{1+\frac{2 \mu}{\lambda}}|\nabla \varphi|^{2}=0
$$

Since $f$ is affine, it vanishes on a set with low dimensionality. We then deduce from the identity (4.8) that $\varphi$ is zero: contradiction.

\section{Appendix: proof of Lemma 3.1}

To show formula (3.4), we observe that if $\boldsymbol{x}$ is not in $\Gamma$, since $\boldsymbol{g}$ is in $C_{c}^{\infty}(\Gamma)$, integrating by parts we can write

$$
\int_{\Gamma}\left(\partial_{y_{1}} \boldsymbol{G}\right)(\boldsymbol{x}, \boldsymbol{y}, \boldsymbol{n}) \boldsymbol{g}(\boldsymbol{y}) d y_{1} d y_{2}=-\int_{\Gamma} \boldsymbol{G}(\boldsymbol{x}, \boldsymbol{y}, \boldsymbol{n})\left(\partial_{y_{1}} \boldsymbol{g}\right)(\boldsymbol{y}) d y_{1} d y_{2},
$$

and then we can apply formula (3.3). We are not aware of formulas (3.5) and (3.6) appearing anywhere in the literature, so we believe that a full proof is called for. By a Taylor expansion,

$$
\boldsymbol{g}\left(y_{1}, y_{2}\right)=\boldsymbol{g}(0,0)+\partial_{y_{1}} \boldsymbol{g}(0,0) y_{1}+\partial_{y_{2}} \boldsymbol{g}(0,0) y_{2}+O\left(\rho^{2}\right),
$$

where $\rho=\sqrt{y_{1}^{2}+y_{2}^{2}}$. Let $R>0$ be small enough so that the circle in the plane $x_{3}=0$ centered at the origin and with radius $R$ is strictly included in $\Gamma$. A long calculation (which we performed thanks to the use of a symbolic computation software), leads to the following expression for $\boldsymbol{G}\left(\boldsymbol{x}, \boldsymbol{y}, \boldsymbol{e}_{1}\right)$ where we only indicate twice the odd $x_{3}$ terms for $x_{1}=x_{2}=0$ : setting

$$
A=\frac{1}{8} \frac{\lambda+3 \mu}{\pi \mu(\lambda+2 \mu)}, \quad B=\frac{1}{8} \frac{\lambda+\mu}{\pi \mu(\lambda+2 \mu)},
$$

$\boldsymbol{G}\left(\boldsymbol{x}, \boldsymbol{y}, \boldsymbol{e}_{1}\right)$ is the product of $\left(\rho^{2}+x_{3}^{2}\right)^{-5 / 2}$ and the matrix whose columns are

$$
\begin{array}{r}
\left(0,0,2\left(\left(\rho^{2}+x_{3}^{2}\right)(A-B) \lambda-2 B \mu\left(x_{3}^{2}-2 y_{1}^{2}+y_{2}^{2}\right)\right) x_{3}\right), \\
\left(0,0,12 \mu B y_{1} y_{2} x_{3}\right), \\
\left(2 x_{3}\left((A-B) x_{3}^{2}+\rho^{2} A+B\left(5 y_{1}^{2}-y_{2}^{2}\right)\right) \mu, 12 \mu B y_{1} y_{2} x_{3}, 0\right) .
\end{array}
$$


We note that for $x_{3}>0$

$$
\int_{0}^{R} \frac{x_{3}^{3} \rho d \rho}{\left(x_{3}^{2}+\rho^{2}\right)^{5 / 2}}=\frac{1}{3} \frac{\left(R^{2}+x_{3}^{2}\right)^{3 / 2}-x_{3}^{3}}{\left(R^{2}+x_{3}^{2}\right)^{3 / 2}}
$$

thus

$$
\lim _{x_{3} \rightarrow 0^{+}} \int_{0}^{2 \pi} \int_{0}^{R} \frac{x_{3}^{3} \rho d \rho d \theta}{\left(x_{3}^{2}+\rho^{2}\right)^{5 / 2}}=\frac{2 \pi}{3} .
$$

Similarly

$$
\lim _{x_{3} \rightarrow 0^{+}} \int_{0}^{2 \pi} \int_{0}^{R} \frac{x_{3} \rho^{3} \cos ^{2} \theta d \rho d \theta}{\left(x_{3}^{2}+\rho^{2}\right)^{5 / 2}}=\frac{2 \pi}{3}
$$

and

$$
\lim _{x_{3} \rightarrow 0^{+}} \int_{0}^{2 \pi} \int_{0}^{R} \frac{x_{3} \rho^{3} \sin ^{2} \theta d \rho d \theta}{\left(x_{3}^{2}+\rho^{2}\right)^{5 / 2}}=\frac{2 \pi}{3}
$$

while by symmetry

$$
\lim _{x_{3} \rightarrow 0^{+}} \int_{0}^{2 \pi} \int_{0}^{R} \frac{x_{3} \rho^{3} \sin \theta \cos \theta d \rho d \theta}{\left(x_{3}^{2}+\rho^{2}\right)^{5 / 2}}=0 .
$$

Thus integrating the matrix $\boldsymbol{G}\left(\boldsymbol{x}, \boldsymbol{y}, \boldsymbol{e}_{1}\right)$ times $\boldsymbol{g}(0,0)$ over the disk in the $x_{1}-x_{2}$ plane with radius $R$ centered at the origin for $x_{3}>0$ and taking the limit as $x_{3}$ approaches zero we find,

$$
\left(\begin{array}{c}
g_{3}(0,0) \\
0 \\
\frac{\lambda}{\lambda+2 \mu} g_{1}(0,0)
\end{array}\right) .
$$

Given that

$$
\lim _{x_{3} \rightarrow 0^{+}} \int_{0}^{2 \pi} \int_{0}^{R} \frac{x_{3}^{3} \rho^{2} d \rho d \theta}{\left(x_{3}^{2}+\rho^{2}\right)^{5 / 2}}=\lim _{x_{3} \rightarrow 0^{+}} \int_{0}^{2 \pi} \int_{0}^{R} \frac{x_{3} \rho^{4} d \rho d \theta}{\left(x_{3}^{2}+\rho^{2}\right)^{5 / 2}}=0,
$$

using Taylor's expansion (5.1), formula (3.5) is proved.

To prove (3.6), we perform another calculation aided by the use of symbolic computation software to find closed form expressions for $\partial_{y_{3}} \boldsymbol{G}\left(\boldsymbol{x}, \boldsymbol{y}, \boldsymbol{e}_{3}\right)$ where, as previously, we only indicate twice the odd $x_{3}$ terms for $x_{1}=x_{2}=0$. It can be written out as the product of

$$
\frac{1}{\left(x_{3}^{2}+\rho^{2}\right)^{7 / 2}}
$$

and the three column vectors

$$
\begin{array}{r}
\left(0,0,-6\left((A+5 B) x_{3}{ }^{2}+\rho^{2}(A-5 B)\right) x_{3} \mu y_{1}\right) \\
\left(0,0,-6\left((A+5 B) x_{3}{ }^{2}+\rho^{2}(A-5 B)\right) x_{3} y_{2} \mu\right) \\
\left(-6 y_{1} x_{3}\left(\left(\rho^{2}+x_{3}{ }^{2}\right)(A-B) \lambda+2 B\left(-3 \rho^{2}+2 x_{3}{ }^{2}\right) \mu\right),\right. \\
\left.-6 y_{2} x_{3}\left(\left(\rho^{2}+x_{3}{ }^{2}\right)(A-B) \lambda+2 B\left(-3 \rho^{2}+2 x_{3}{ }^{2}\right) \mu\right), 0\right)
\end{array}
$$


Clearly, by symmetry,

$$
\int_{0}^{2 \pi} \int_{0}^{R} \frac{y_{j} x_{3} \rho^{3} d \rho d \theta}{\left(x_{3}^{2}+\rho^{2}\right)^{7 / 2}}=\int_{0}^{2 \pi} \int_{0}^{R} \frac{y_{j} x_{3}^{3} \rho d \rho d \theta}{\left(x_{3}^{2}+\rho^{2}\right)^{7 / 2}}=0
$$

for $j=1$ or 2 . Thus there will be no contribution from $\boldsymbol{g}(0,0)$. Similarly, the cross terms

$$
\int_{0}^{2 \pi} \int_{0}^{R} \frac{y_{j} y_{k} x_{3} \rho^{3} d \rho d \theta}{\left(x_{3}^{2}+\rho^{2}\right)^{7 / 2}}=\int_{0}^{2 \pi} \int_{0}^{R} \frac{y_{j} y_{k} x_{3}^{3} \rho d \rho d \theta}{\left(x_{3}^{2}+\rho^{2}\right)^{7 / 2}}=0
$$

are zero if $j \neq k$. Now a calculation will show that the following limits hold,

$$
\begin{aligned}
& \lim _{x_{3} \rightarrow 0^{+}} \int_{0}^{2 \pi} \int_{0}^{R} \frac{x_{3} \rho^{5} \cos ^{2} \theta d \rho d \theta}{\left(x_{3}^{2}+\rho^{2}\right)^{7 / 2}}=\lim _{x_{3} \rightarrow 0^{+}} \int_{0}^{2 \pi} \int_{0}^{R} \frac{x_{3} \rho^{5} \sin ^{2} \theta d \rho d \theta}{\left(x_{3}^{2}+\rho^{2}\right)^{7 / 2}}=\frac{8 \pi}{15} \\
& \lim _{x_{3} \rightarrow 0^{+}} \int_{0}^{2 \pi} \int_{0}^{R} \frac{x_{3}^{3} \rho^{3} \cos ^{2} \theta d \rho d \theta}{\left(x_{3}^{2}+\rho^{2}\right)^{7 / 2}}=\lim _{x_{3} \rightarrow 0^{+}} \int_{0}^{2 \pi} \int_{0}^{R} \frac{x_{3}^{3} \rho^{3} \sin ^{2} \theta d \rho d \theta}{\left(x_{3}^{2}+\rho^{2}\right)^{7 / 2}}=\frac{2 \pi}{15}
\end{aligned}
$$

We then combine (5.75.8 5.11) with Taylor formula (5.1) to find a contribution of $\frac{\lambda}{\lambda+2 \mu}\left(\partial_{y_{1}} \boldsymbol{g}(0,0)+\right.$ $\left.\partial_{y_{2}} \boldsymbol{g}(0,0)\right)$ in the direction of $\boldsymbol{e}_{3}$, and $\nabla_{\Gamma} g_{3}(0,0)$ in the $\boldsymbol{e}_{1}, \boldsymbol{e}_{2}$ plane. Higher order terms won't contribute since

$$
\lim _{x_{3} \rightarrow 0^{+}} \int_{0}^{R} \frac{x_{3} \rho^{6} d \rho}{\left(x_{3}^{2}+\rho^{2}\right)^{7 / 2}}=\lim _{x_{3} \rightarrow 0^{+}} \int_{0}^{R} \frac{x_{3}^{3} \rho^{4} d \rho}{\left(x_{3}^{2}+\rho^{2}\right)^{7 / 2}}=0,
$$

and formula (3.6) is proved.

Formula (3.7) is derived likewise.

To prove formula (3.8) we need a higher order Taylor formula. We write,

$$
\begin{array}{r}
\boldsymbol{g}\left(y_{1}, y_{2}\right)=\boldsymbol{g}(0,0)+\partial_{y_{1}} \boldsymbol{g}(0,0) y_{1}+\partial_{y_{2}} \boldsymbol{g}(0,0) y_{2} \\
+\frac{1}{2} \partial_{y_{1}}^{2} \boldsymbol{g}(0,0) y_{1}^{2}+\frac{1}{2} \partial_{y_{2}}^{2} \boldsymbol{g}(0,0) y_{2}^{2}+\partial_{y_{1}} \partial_{y_{2}} \boldsymbol{g}(0,0) y_{1} y_{2}+O\left(\rho^{3}\right) .
\end{array}
$$

We perform another calculation aided by the use of symbolic computation software to find expressions for $\partial_{x_{3}} \partial_{y_{3}} \boldsymbol{G}\left(\boldsymbol{x}, \boldsymbol{y}, \boldsymbol{e}_{3}\right)$. For the sake of brevity we only give a proof in the case where $g_{3}=0$, so we only need the first two columns. We only indicate twice the odd $x_{3}$ terms for $x_{1}=x_{2}=0$. They are the the product of $\frac{1}{\left(\rho^{2}+x_{3}^{2}\right)^{\frac{9}{2}}}$ and a matrix whose first column is

$$
\begin{array}{r}
6 \mu\left(2 x_{3}{ }^{4}-\left(41{y_{1}}^{2}+y_{2}{ }^{2}\right) x_{3}{ }^{2}+3 \rho^{2}\left(9 y_{1}{ }^{2}-y_{2}{ }^{2}\right)\right) x_{3} B+6\left(3 \rho^{4}+\rho^{2} x_{3}{ }^{2}-2 x_{3}{ }^{4}\right) \mu x_{3} A \\
-60 y_{1} y_{2}\left(-3 \rho^{2}+4 x_{3}{ }^{2}\right) B x_{3} \mu
\end{array}
$$

and whose second column is

$$
\begin{aligned}
& -60 y_{1} y_{2}\left(-3 \rho^{2}+4 x_{3}^{2}\right) B x_{3} \mu \\
& 6 \mu\left(2 x_{3}{ }^{4}-\left(41 y_{1}{ }^{2}+y_{2}{ }^{2}\right) x_{3}{ }^{2}+3 \rho^{2}\left(9 y_{1}{ }^{2}-y_{2}{ }^{2}\right)\right) x_{3} B+6\left(3 \rho^{4}+\rho^{2} x_{3}{ }^{2}-2 x_{3}{ }^{4}\right) \mu x_{3} A
\end{aligned}
$$


Further calculations show that if we multiply these two columns by $\frac{\rho}{\left(\rho^{2}+x_{3}^{2}\right)^{\frac{9}{2}}}$ integrate in $\rho$ from 0 to $R$ and and $\theta$ from 0 to $2 \pi$ and then take the limit as $x_{3}$ tends to zero, we find zero. We also find zero as we multiply by $\frac{\rho^{2} \cos \theta}{\left(\rho^{2}+x_{3}^{2}\right)^{\frac{9}{2}}}, \frac{\rho^{2} \sin \theta}{\left(\rho^{2}+x_{3}^{2}\right)^{\frac{9}{2}}}$, or by $\frac{\rho^{p}}{\left(\rho^{2}+x_{3}^{2}\right)^{\frac{9}{2}}}$, if $p \geq 4$. We only find non-zero contributions (which are independent of $R>0$ ) for the second derivatives of $\boldsymbol{g}$ : they are found by multiplying by $\frac{\rho^{3} \sin ^{p} \theta \cos ^{q} \theta}{\left(\rho^{2}+x_{3}^{2}\right)^{\frac{9}{2}}}$, where $p, q$ are in $\{0,1,2\}$ with $p+q=2$. More precisely, for the $\partial_{1}^{2}$ derivative, we find, for the first column

$$
\left(\frac{6 \lambda+8 \mu}{\lambda+2 \mu}, 0,0\right),
$$

for the second column

$$
(0,2,0)
$$

For the $\partial_{2}^{2}$ derivative, we find, for the first column

$$
(2,0,0)
$$

for the second column

$$
\left(0, \frac{6 \lambda+8 \mu}{\lambda+2 \mu}, 0\right) .
$$

For the $\partial_{1} \partial_{2}$ derivative, we find, for the first column

$$
\left(0, \frac{2 \lambda+2 \mu}{\lambda+2 \mu}, 0\right)
$$

for the second column

$$
\left(\frac{2 \lambda+2 \mu}{\lambda+2 \mu}, 0,0\right) .
$$

Finally, we arrive at jump formula (3.8) thanks to a linear combination.

The same proof technique is used for deriving formula (3.9). For the sake of brevity, here too we assume $g_{3}=0$. This time a Taylor expansion of order 1 is sufficient. The first two columns of $\partial_{x_{3}} \boldsymbol{G}\left(\boldsymbol{x}, \boldsymbol{y}, \boldsymbol{e}_{1}\right)$ where we only indicate twice the odd $x_{3}$ terms for $x_{1}=x_{2}=0$ are is the product of $\frac{1}{\left(x_{3}^{2}+\rho^{2}\right)^{7 / 2}}$ and

$$
\begin{array}{r}
6 x_{3} y_{1}\left(\left(\rho^{2}+x_{3}{ }^{2}\right)(A-B) \lambda+\mu\left(2 A\left(\rho^{2}+x_{3}{ }^{2}\right)-2 B\left(2 x_{3}{ }^{2}-3 y_{1}{ }^{2}+2 y_{2}{ }^{2}\right)\right)\right) \\
6 x_{3} y_{2}\left(\left(\rho^{2}+x_{3}{ }^{2}\right)(A-B) \lambda-2 B \mu\left(x_{3}{ }^{2}-4 y_{1}{ }^{2}+y_{2}{ }^{2}\right)\right)
\end{array}
$$

and

$$
\begin{aligned}
& 6 \mu x_{3} y_{2}\left((A-B) y_{2}^{2}+(A+9 B) y_{1}^{2}+x_{3}^{2}(A-B)\right) \\
& 6 \mu x_{3} y_{1}\left(x_{3}^{2}(A-B)+(A-B) y_{1}{ }^{2}+y_{2}{ }^{2}(A+9 B)\right)
\end{aligned}
$$

We then multiply (5.14) and (5.17) by $\frac{\rho y_{i}}{\left(\rho^{2}+x_{3}^{2}\right)^{\frac{7}{2}}}, i=1,2$ and integrate in $\rho$ from 0 to $R$, and $\theta$ from 0 to $2 \pi$ to only find non-zero contributions (which are independent of $R>0$ ) for the first derivatives of $\boldsymbol{g}$. After simplification, we obtain formula (3.9). 


\section{Conclusion}

In this paper we studied the well-posedness of the fault inverse problem. We derived stability estimates for determining the plane containing the fault. We proved that if the slip field is known, this determination is Lipschitz stable. In the more realistic case where the slip field is unknown, we showed another Lipschitz stability result under the additional assumption, which seems physically relevant, that the slip field is one directional. The proofs of the results presented in this paper are non-constructive and thus provide no insight on how the stability constants depend on the physics and on the geometry of the problem. This will be the subject of forthcoming work.

\section{Acknowledgements}

D. Volkov is supported by a Simons Foundation Collaboration Grant. The research of FT was supported in part by the grant ANR-17-CE40-0029 of the French National Research Agency ANR (project MultiOnde), and the LabEx PERSYVAL-Lab (ANR-11-LABX- 0025-01).

\section{References}

[1] title=Classe di Scienze, author=Alessandrini, Giovanni and Beretta, Elena and Rosset, Edi and Vessella, Sergio, journal=Ann. Scuola Norm. Sup. Pisa Cl. Sci.(4), volume=29, pages $=755-806$, year $=2000$

[2] title=Identification of an inclusion in multifrequency electric impedance tomography, author $=$ Ammari, Habib and Triki, Faouzi, journal=Communications in Partial Differential Equations, volume $=42$, number $=1$, pages $=159-177$, year $=2017$, publisher $=$ Taylor \& Francis

[3] , title=The 1989 Loma Prieta earthquake imaged from inversion of geodetic data, author=Arnadóttir, Thóra and Segall, Paul, journal=Journal of Geophysical Research: Solid Earth (1978-2012), volume=99, number $=$ B11, pages $=21835-21855$, year $=1994$, publisher $=$ Wiley Online Library

[4] title $=$ Determination of a linear crack in an elastic body from boundary measurementsLipschitz stability, author=Beretta, Elena and Francini, Elisa and Vessella, Sergio, journal=SIAM Journal on Mathematical Analysis, volume $=40$, number $=3$, pages $=984-1002$, year $=2008$, publisher $=$ SIAM

[5] AUTHOR = O. P. Cavalié and M. Vergnolle and M. Cotte, TITLE = Slow slip event in the Mexican subduction zone: evidence of shallower slip in the Guerrero seismic gap for the 2006 event revealed by the joint inversion of InSAR and GPS data, JOURNAL $=$ Earth and Planetary Science Letters, VOLUME $=367$, YEAR $=2013$,

[6] $\mathrm{AUTHOR}=\mathrm{H}$. Dragert and K. L. Wang and T. S. James, TITLE = A silent slip event on the deeper Cascadia subduction interface, JOURNAL $=$ Science, VOLUME $=5521$, $\mathrm{YEAR}=2001, \mathrm{PAGES}=1525-1528$ 
[7] title=Determining cracks by boundary measurements, author=Friedman, Avner and Vogelius, Michael, year=1989, note=http://conservancy.umn.edu/bitstream/handle/11299/4926/476.pdf

[8] title=Repeated large slow slip events at the southcentral Alaska subduction zone, author $=\mathrm{Fu}$, Yuning and Freymueller, Jeffrey T, journal=Earth and Planetary Science Letters, volume $=375$, pages $=303-311$, year $=2013$, publisher $=$ Elsevier

[9] title=A non-linear geodetic data inversion using ABIC for slip distribution on a fault with an unknown dip angle, author=Fukahata, Yukitoshi and Wright, Tim J, journal=Geophysical Journal International, volume $=173$, number $=2$, pages $=353-364$, year $=2008$, publisher $=$ Oxford University Press

[10] $\mathrm{AUTHOR}=$ A. Guilhem and R. M. Nadeau, TITLE = Episodic tremors and deep slowslip events in Central California, JOURNAL = Earth and Planetary Science Letters, $\mathrm{VOLUME}=357, \mathrm{YEAR}=2012, \mathrm{PAGES}=1-10$

[11] , title=Fault slip distribution of the $1999 \mathrm{M} \mathrm{w} 7.1$ Hector Mine, California, earthquake, estimated from satellite radar and GPS measurements author=Jónsson, Sigurjón and Zebker, Howard and Segall, Paul and Amelung, Falk, journal=Bulletin of the Seismological Society of America, volume=92, number=4, pages=1377-1389, year=2002, publisher $=$ Seismological Society of America

[12] title=Perturbation theory for linear operators, author=Kato, Tosio, volume=132, year $=2013$, publisher $=$ Springer Science \& Business Media

[13] AUTHOR $=$ Y. Kim and R. W. Clayton and J. M. Jackson, TITLE = Geometry and seismic properties of the subducting Cocos plate in central Mexico, JOURNAL $=\mathrm{J}$. Geophys. Res., VOLUME $=115$, YEAR $=2010$, PAGES $=$

[14] AUTHOR $=$ Y. Okada, TITLE = Internal deformation due to shear and tensile faults in a half-space, JOURNAL = Bulletin of the Seismological Society of America, VOLUME $=$ vol. 82 no. 2 , YEAR $=1992$, PAGES $=1018-1040$

[15] AUTHOR = M. Radiguet and F. Cotton and M. Vergnolle and M. Campillo and B. Valette and V. Kostoglodov and N. Cotte, TITLE = Spatial and temporal evolution of a long term slow slip event: the 2006 Guerrero Slow Slip Event, JOURNAL = Geophysical Journal International, VOLUME $=$, YEAR $=2010$, PAGES =

[16] AUTHOR = M. Radiguet and F. Cotton and M. Vergnolle and M. Campillo and B. Valette and V. Kostoglodov and N. Cotte, TITLE = Spatial and temporal evolution of a long term slow slip event: the 2006 Guerrero Slow Slip Event, JOURNAL = Geophysical Journal International, VOLUME $=184(2)$, YEAR $=2011$, PAGES $=816-828$

[17] title=Inverse problem theory and methods for model parameter estimation, author $=$ Tarantola, Albert, year $=2005$, publisher $=$ siam

[18] AUTHOR = D. Volkov, TITLE = A Double Layer Surface Traction Free Green's Tensor, JOURNAL = SIAM J. APPL. MATH., VOLUME = 69 (5), YEAR = 2009, PAGES $=1438-1456$ 
[19] title=Determining Fault Geometries From Surface Displacements, author=Volkov, D. and Voisin, C. and I.R., Ionescu, journal=Pure and Applied Geophysics, volume $=174$, number $=4$, pages $=1659-1678$, year $=2017$, publisher $=$ Springer

[20] title $=$ An eigenvalue problem for elastic cracks in free space, author=Volkov, Darko, journal=Mathematical Methods in the Applied Sciences, volume $=33$, number $=5$, pages $=607-622$, year $=2010$, publisher $=$ Wiley Online Library

[21] title $=\mathrm{A}$ stochastic approach to reconstruction of faults in elastic half space, author=Volkov, Darko and Sandiumenge, Joan Calafell, journal=arXiv preprint arXiv:1709.03614, year $=2017$

[22] title=Reconstruction of faults in elastic half space from surface measurements, author=Volkov, Darko and Voisin, Christophe and Ionescu, Ioan, journal=Inverse Problems, volume $=33$, number $=5$, year $=2017$ 